\title{
Genomic analysis of Landes goose origin goose hemorrhagic polyomavirus, China
}

\author{
Ling $\mathrm{Luo}^{1}$, Xinglong $\mathrm{Yu}^{1}$, Xiang $\mathrm{Qu}^{2}$, Fei Zhao ${ }^{1}$, Yan Ding $^{1}$, Dun Zhao ${ }^{1}$, Yan Xiang ${ }^{1}$, Zai \\ $\mathrm{Mao}^{1}$, Nuo $\mathrm{Li}^{1}$, and Lei Yang${ }^{1}$ \\ ${ }^{1}$ Hunan Agricultural University \\ ${ }^{2}$ Hunan Agricultural University College of Animal Science and Technology
}

September 24, 2021

\begin{abstract}
A goose hemorrhagic polyomavirus (GHPV) outbreak occurred in a Goose Farm in Hunan, China, between January and July 2021. Approximately 1,500 breeding goose died, and hatching rates dropped from the previous $85 \%$ to about $50 \%$ in this outbreak. GHPV HUN-01, isolated from the liver of infected Landes geese, shared a close evolutionary relationship with the Toulouse Goose 2000 and 14234 strain, isolated from geese in France and Hungary. The isolation of GHPV from the livers of dead embryos also demonstrates that the virus can be transmitted vertically. In conclusion, clinical and laboratory diagnostics of the diseased geese in this outbreak were consistent with GHPV being the causative agent. We learned that this is the first time that GHPV has been isolated from geese in mainland China.
\end{abstract}

\section{Genomic analysis of Landes goose origin goose hemorrhagic polyomavirus, China}

Lingzhi Luo ${ }^{1}$, XingLong $\mathrm{Yu}^{1}$ XiangYong $\mathrm{Qu}^{2}$,FeiYan Zhao ${ }^{1}$, YanBin Ding ${ }^{1}$,Dun Zhao ${ }^{1}$, YanHui Xiang ${ }^{1}$, ZaiYang $\mathrm{Mao}^{1}$, Nuo $\mathrm{Li}^{1}$, Lei Yang ${ }^{1 *}$

${ }^{1}$ College of Veterinary Medicine, Hunan Agricultural University, Changsha, China

${ }^{2}$ College of Animal Science and Technology,Hunan Agricultural University, Changsha, China

*Correspondence should be addressed to Lei Yang; 277670218@qq.com

\section{Summary}

A goose hemorrhagic polyomavirus (GHPV) outbreak occurred in a Goose Farm in Hunan, China, between January and July 2021. Approximately 1,500 breeding goose died, and hatching rates dropped from the previous $85 \%$ to about $50 \%$ in this outbreak. GHPV HUN-01, isolated from the liver of infected Landes geese, shared a close evolutionary relationship with the Toulouse Goose 2000 and 14234 strain, isolated from geese in France and Hungary. The isolation of GHPV from the livers of dead embryos also demonstrates that the virus can be transmitted vertically. In conclusion, clinical and laboratory diagnostics of the diseased geese in this outbreak were consistent with GHPV being the causative agent. We learned that this is the first time that GHPV has been isolated from geese in mainland China.

KEYWORDS: Goose; GHPV;Genomic analysis

\section{INTRODUCTION}

Hemorrhagic nephritis and enteritis, a high morbidity and mortality disease of geese, was first described in 1969 in Hungary (Bernath \& Szalai, 1970). A few years later it occurred in France(Schettler, 1977; Vuillaume et al.,1982), Germany(Schettler, 1980), Poland(Gawet et al.,2014;Kozdrun et al.,2012) and in 
Belgium (Garmyn et al.,2017). The causative dsDNA virus, the goose hemorrhagic polyomavirus(GHPV), or recently named Anser anser polyomavirus 1,belongs to the Gammapolyomavirus genus of the Polyomaviridae family(Guerin et al., 2000). It was previously reported that one-day-old goslings can reproduce GHPV by non-oral route infection; goslings are susceptible to subcutaneous or intraperitoneal inoculation; GHPV can also be reproduced through goose embryos, 8-10 after inoculation of 14-day-old goose embryos through chorioallantoic membrane Death after days, the disease is similar to the infection of 1-day-old goose embryos. It can be passaged by culture of 1-day-old goose kidney epithelial cells. Cytopathies (CPE), cytoplasmic granulation and vacuoles appear after 5 days of inoculation, and then the virus Sprout and release from the cell.( Bernath, S et al.,2006)The genome of GHPV is 5252-5256 bp long and comprises the genes of the VP1-VP3 structure proteins, the large and small tumor antigens (LTA and STA), and the predicted ORF-X with unknown function (Feher et al.,2014; Johne \& Miller, 2003). GHPV is known to cause disease in geese but may also replicate in other bird species without causing clinical symptoms (Corrand et al., 2011; Pingret et al., 2008). The infection affects the internal organs via tropism for endothelial cells, resulting in vascular dysfunctions, edema, ascites, hemorrhages, enteritis and neurological signs before death of young geese(Bernath \& Szalai, 1970; Dobos-Kovacs et al., 2005; Guerin et al., 2000; Lacroux et al., 2004).GHPV may also persist in domestic waterfowl farms without any signs of disease(Chunhe et al., 2018; Garmyn et al.,2017; Kozdrun et al., 2012; Leon et al.,2013;Palya et al., 2004; Wan et al., 2018). GHPV constitute a genetically distinct gammapolyomavirus with a maximum of $69 \%$ nt identity compared to the genome of other avian polyomaviruses. Similarly to other polyomaviruses its genome seems to be highly conserved with genes typical for the genus(Kaszab, Eet al.,2020; Tu, Y. Cet al.,2021).

\section{MATERIALS AND METHODS}

\subsection{Herd history and case presentation}

Since 2017, there have been reports of large-scale deaths of imported species, mainly Landes' geese, all over the country. Among them, the responses of farmers in Shandong, Hebei, Anhui, Yunnan, Jiangsu and Hunan are the most prominent. Related reports since 2018 show that the isolated viruses are mainly astrovirus, followed by goose circovirus, and there are also mixed feelings of the two. With the use of yolk antibodies, astrovirus infection was controlled. However, there are still goose farms reporting that goose deaths have occurred. Therefore, we realize that the emergence of this phenomenon should not only be caused by astrovirus and circovirus infection, but there should be undiscovered potential pathogens. Combining clinical signs and applying molecular biology methods, we isolated another potential virus, goose hemorrhagic polyomavirus (GHPV), at the Landers goose breeding site in Hunan, China.

\subsection{Pathogen examination}

Tissues, including the cerebral cortex, Kidney, liver, spleen, intestine and lung, were collected from six Landes geese to diagnose the pathogen in the infected geese.Viral RNA and DNA were extracted using nucleic acid extraction kits (Accurate biology, Hunan, China).Viral cDNA was synthesized using the RevertAid FirstStrand cDNA Synthesis Kit (Thermo Scientific, Waltham, MA, USA)according to the manufacturer's instructions. The cDNA and DNA were subjected to PCR to detect a panel of potential viral pathogens, including Goose parvovirus (GPV), goose hemorrhagic polyomavirus (GHPV), goose reovirus (GRV), goose paramyxovirus (GPMV), duck tambusu Virus (TMUV), and astrovirus(AstV)(The detection primers are in the Table S1).cerebral cortex, Kidney, liver, spleen, intestine and lung tissues were also submitted for bacterial culture.

\subsection{Isolation and genetic analysis of the virus}

The supernatant of each GHPV positive sample was filtered first through $0.45 \mu \mathrm{m}$ filters (Pall Corporation Syringe Filter, Germany) then through $0.22 \mu \mathrm{m}$ filters,and the final filtrate was used for virus isolation as previously described. Each sample used six specific pathogen-free (SPF) Landes Goose embryos (14-day) for virus isolation. Each inoculated embryo was incubated at 37 for 8-10 day, candling of the inoculated embryos every $12 \mathrm{~h}$. 
A GHPV strain isolated from the cerebrumofan infected Landes gooses was selected for commercial whole genome sequencing (Tsingke, Beijing, China) using the primer pairs shown in Table S1 and the pClone007 Blunt Simple Vector Kit (Tsingke, Beijing, China). Sequences were analysed with the Lasergene package (DNASTAR, Madison, WI, USA)( The CHPV Sequencing are in the Table S2). Genomic comparative research was undertaken by using the ClustalW method for pairwise comparison. Previously reported GHPV genomes were retrieved from GenBank, and the information for each of the GHPVs and Another the eight Polyomaviridae famil are listed in Table 1.To determine the relationships among these viruses, genome and LTAg protein sequences were aligned in ClustalW, as implemented in MEGA7.0. Phylogenies were reconstructed in MEGA7.0 using the maximum likelihood and neighbor joining methods with 1,000 bootstrap pseudo-replicates. (Tamura, Stecher, Peterson,Filipski\&Kumar,2013).

\subsection{Distribution of the virus in organs and pathological examination}

While six infected geese were randomly selected for pathological autopsy, tissues of cerebral cortex, kidney, liver, heart, lung and spleen were collected for GHPV PCR testing in order to understand the distribution of GHPV in the major organs of infected Langdes geese.

For naturally dead embryos between 14 and 21 days of age, chorionic villous fluid and major embryonic organs (liver, kidney, heart and spleen) were collected for GHPV PCR testing along with pathological dissection of the embryos.

The isolated GHPV was inoculated into 14-day-old healthy goose embryos via the chorionic villus allantoic membrane. The embryos were pathologically dissected and the chorionic allantoic fluid and the main organs of the embryos (liver, kidney, heart and spleen) were collected for GHPV PCR assay. To clarify the distribution of the virus after inoculation.

\section{RESULTS AND DISCUSSION}

\subsection{Pathogen examination}

All the tested samples from two diseased Landes geese, examined for the presence of GPV, GRV, GPMV, TMUV and AstV, were negative. Cerebraland lung tissues, submit-ted for bacterial culture, were also negative for bacterial pathogens.

\subsection{Isolation and genetic analysis of the virus}

The allantoic fluid and embryo bodies of SPF goose embryos that were inoculated with diseased material and cultured for 8-10 day were tested by PCR for goose polyoma virus pathogen. It was found that the allantoic fluid PCR test result was negative, and the embryo body test result was weakly positive.

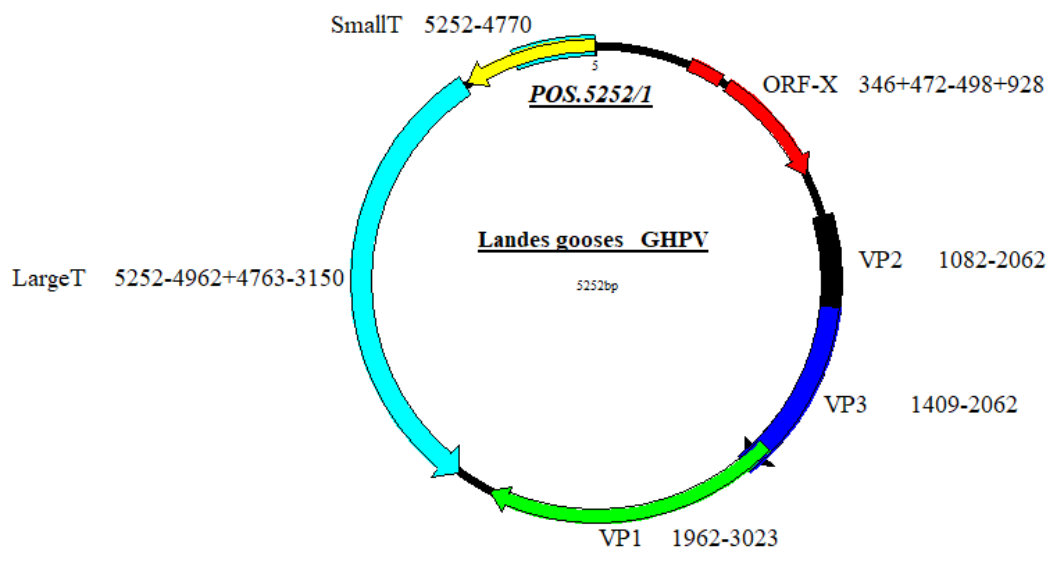

FIGURE 1 
After assembly with the Lasergene package, the Landes Goose origin GHPV (strains HUN-01) genomes were obtained and submitted to the GenBank under accession Nos. MZ614863. The genome of Landes Goose origin GHPV (HUN-01 strains) was found to be 5252 nucleotides in length. The genome length of goose origin GHPV in China was $5252 \mathrm{bp}$, whereas the strains from France and Germany had a genome length range of 5252 to $5256 \mathrm{bp}$, respectively (Table 1). All of the GHPVs had the same coding regions (six ORFs) in length, including the ORF-X, VP2, VP3, VP1, LTAg, and STAg. The putative ORF-X protein encodes 120 amino acid (aa; positions 347-424 plus 497-928); VP2 encodes 211 aa (position 1082-2062); VP3 shares the same terminal as VP2 and encodes 139 aa (position 1409-2062);VP1 encodes 237 aa (position 19623023);LTAg encodes 397 aa (positions 3150- 4763 plus 4956-5252); and STAg encodes 100 aa (position 4770-5252). The locations of the protein coding regions are shown in FIGURE 1 .

Table 1 . Information of the polyomaviruses strains used in this study

\begin{tabular}{lllll}
\hline GenBank accession No. & Strain & Host & Length (bp) & Area \\
\hline AY140894 & Germany 2001 & Goose & 5256 & Germany \\
HQ681902 & Toulouse Goose 2008 & Goose & 5254 & France \\
HQ681903 & Toulouse Goose 2000 & Goose & 5252 & France \\
HQ681904 & Toulouse Muscovy Duck 2008 & Muscovy duck & 5254 & France \\
HQ681905 & Toulouse Mule Duck 2008 & Mule duck & 5253 & France \\
JF304775 & 106 & Pekin duck & 5254 & China \\
KJ452212 & 14234 & Goose & 5252 & Hungary \\
MG190356 & JY140Ma & Sheldrake & 5254 & China \\
MG670535 & JY141Ma & Sheldrake & 5254 & China \\
$M$ Z614863 & GHPV-HUN-01 & Landes gooses & 5252 & China \\
AF241168 & Budgerigar fledgling disease virus -1 & budgerigar & 4981 & Unknow \\
AF442959 & Murine polyomavirus1 & mouse & 5307 & England \\
AY672646 & BFPyV-1 & budgerigar & 4981 & China \\
DQ192570 & Corvus monedula polyomavirus 1 & Eurasian jackdaw & 5079 & Germany \\
DQ192571 & Pyrrhula pyrrhula polyomavirus 1 & Eurasian bullfinch & 5278 & Germany \\
GU345044 & CaPyV-Ha09 & Serinus canaria & 5421 & Netherlands \\
HM011560 & 607a & Homo sapiens & 4926 & USA \\
J02400 & Simian virus 40 & rhesus monkey & 5243 & Unknown \\
\hline
\end{tabular}



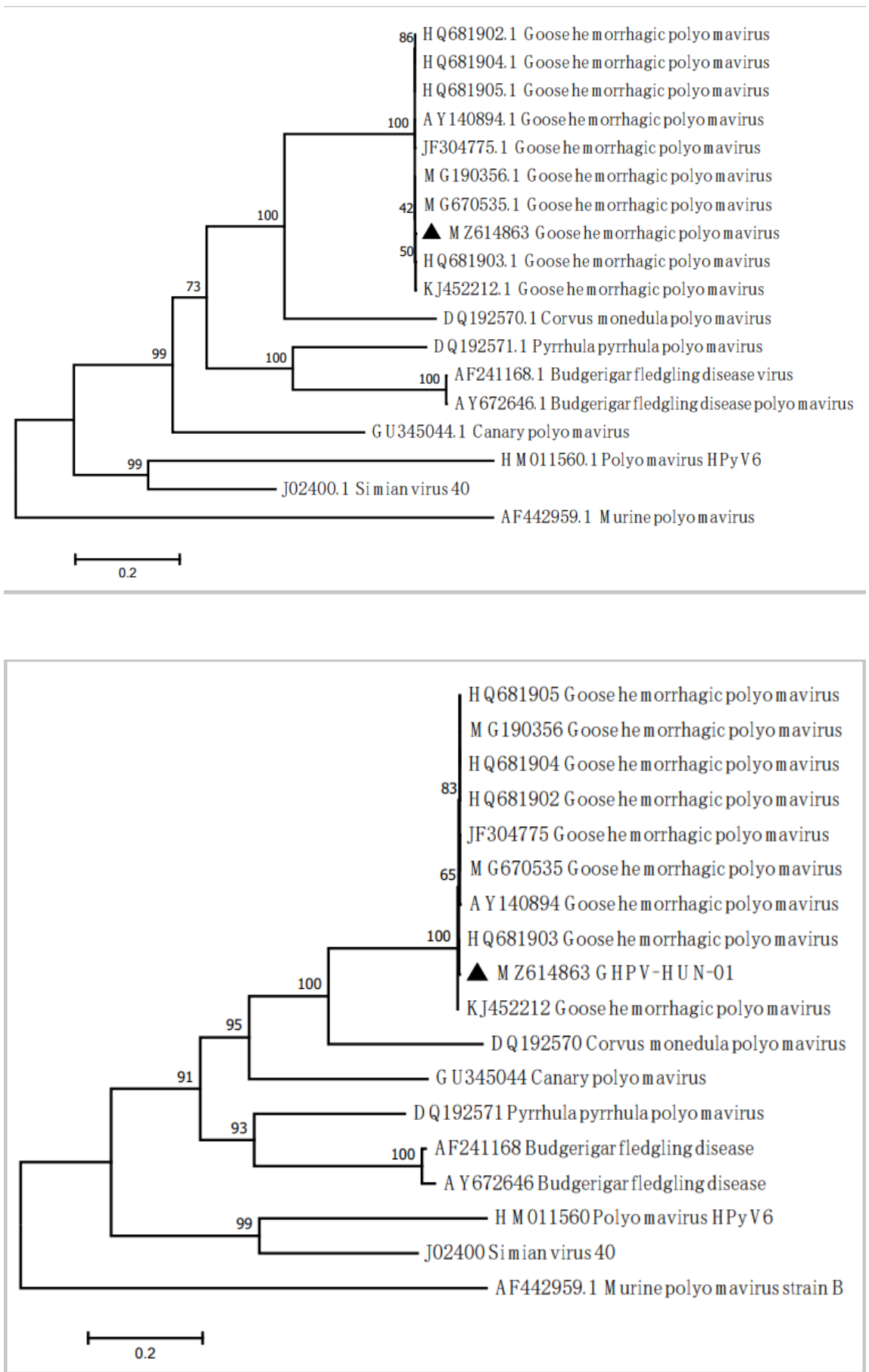

FIGURE 2 Evolutionary relationships. Evolutionary history was inferred using.

The maximum likelihood method with the Tamura-Nei model and gamma-distributedrate heterogeneity in MEGA 7.0. The percentage of replicates in which the associated virus clustered together in the bootstrap test (1,000 replicates) is shown next to the branches in each tree. The scale barindicates nucleotide substitutions per site. The strain isolated in this study is identified by (a)Evolutionarytreebasedonthenucleotidesequencesofthebasedonthealignmentofgenome.(b)Evolutionarytreebasedonther 


\subsection{Distribution of the virus in organs, and pathological examination}

The main pathological changes were: emaciation and marked fecal contamination around the cloaca. Liver: uneven color, swollen, brittle. Some sick geese can be seen in the liver lobe edge gray-white lesion area; kidney: swollen, flushed color, brittle and fragile texture. The goose with a long course of disease can be seen on the surface of the kidney with gray-white urate deposits; intestine: 12 fingers of intestine, jejunum mucosa flushed, intestinal cavity can be seen in a large amount of blood-like contents; pancreas: swollen, the color is earthy yellow.

Histopathological findings showed that in the liver: focal infiltration of mononuclear cells in the hepatic lobules (a) and confluent area (b), widening of the sinusoidal gap due to amyloid deposition, and loss of the intrinsic hepatic cord structure. Heart (c): Focal infiltration of inflammatory cells such as lymphocytes and macrophages around the injured myocardial fibers. Kidney: significant hemorrhage (d) and extensive necrosis of renal tubular $(\mathrm{e}, \mathrm{f})$ epithelial cells. In other organs, there were no obvious histological lesions.
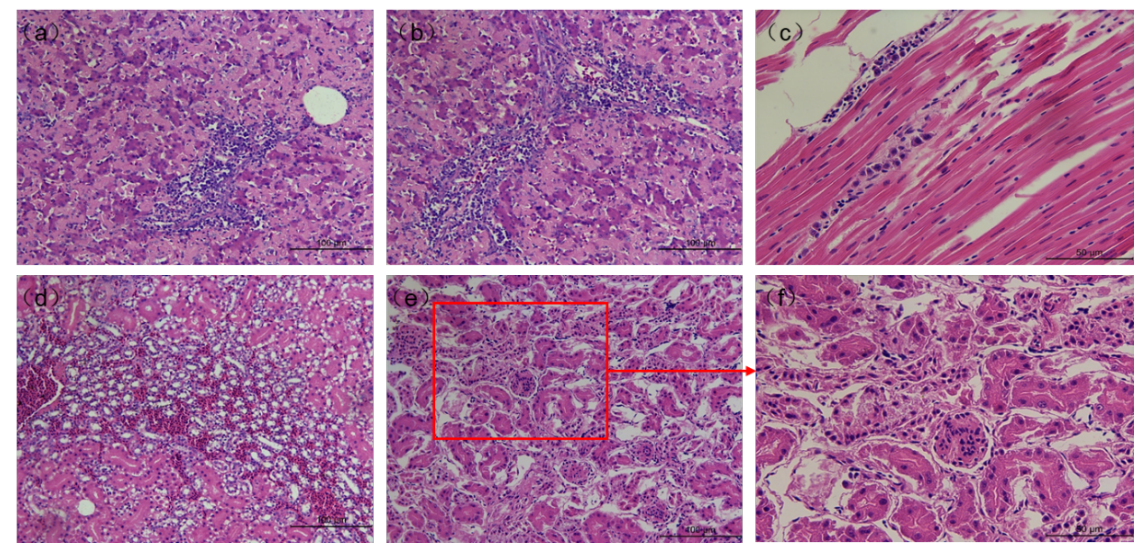

FIGURE 3 Histopathological analysis (haematoxylin and eosin staining) of tissues from a dead goose infected with GHPV. (a) A pale pink amyloid deposition in the hepatic sinusoid widens the sinusoidal gap, and a focal infiltrate of lymphocyte-dominated mononuclear cells is seen next to the central vein. (original magnification 200x). Scale bar indicates $100 \mu \mathrm{m}$. (b) Lymphocyte-dominated mononuclear cells show peritubular infiltration in the confluent area between liver lobules. (original magnification 200x). Scale bar indicates $100 \mu \mathrm{m}$. (c) Myocardial fibers were broken and lysed, and focal infiltration of inflammatory cells dominated by lymphocytes and macrophages was seen in the interstitium. (original magnification 400x). Scale bar indicates $50 \mu \mathrm{m}$.(d) Erythrocytes flow out of the blood vessels and fill the renal interstitium. (original magnification 200x). Scale bar indicates $100 \mu \mathrm{m}$. (e) Large tubular necrosis. (original magnification 200x). Scale bar indicates $100 \mu \mathrm{m}$. (f) Renal tissues with lesions of renal tubular epithelial cells exhibited varying degrees of nuclear necrosis of renal tubular epithelial cells. (original magnification 400x). Scale bar indicates $50 \mu \mathrm{m}$.

The results of PCR testing of the viruses in each organ of GHPV-infected geese showed that the viruses were distributed in the liver, spleen, heart, lung and kidney, and were mainly distributed in the liver and spleen.

From February 2021 to the present, the hatching rate of female geese in this goose farm decreased significantly (from $85 \%$ to 50\%), and eggs laid by a female goose infected with GHPV will hatch until about 21 days, at which point the embryo will die. The pathological examination of the dead embryos revealed that the embryonic liver showed obvious lesions: some geese embryonic liver was grayish yellow, swollen, and a few red spots were visible at the edge of the liver lobe (FIGURE 4a ); some geese embryonic liver was unevenly colored, swollen, and visible as diffusely distributed grayish white Small rice-grain sized lesions (FIGURE $4 b)$. 

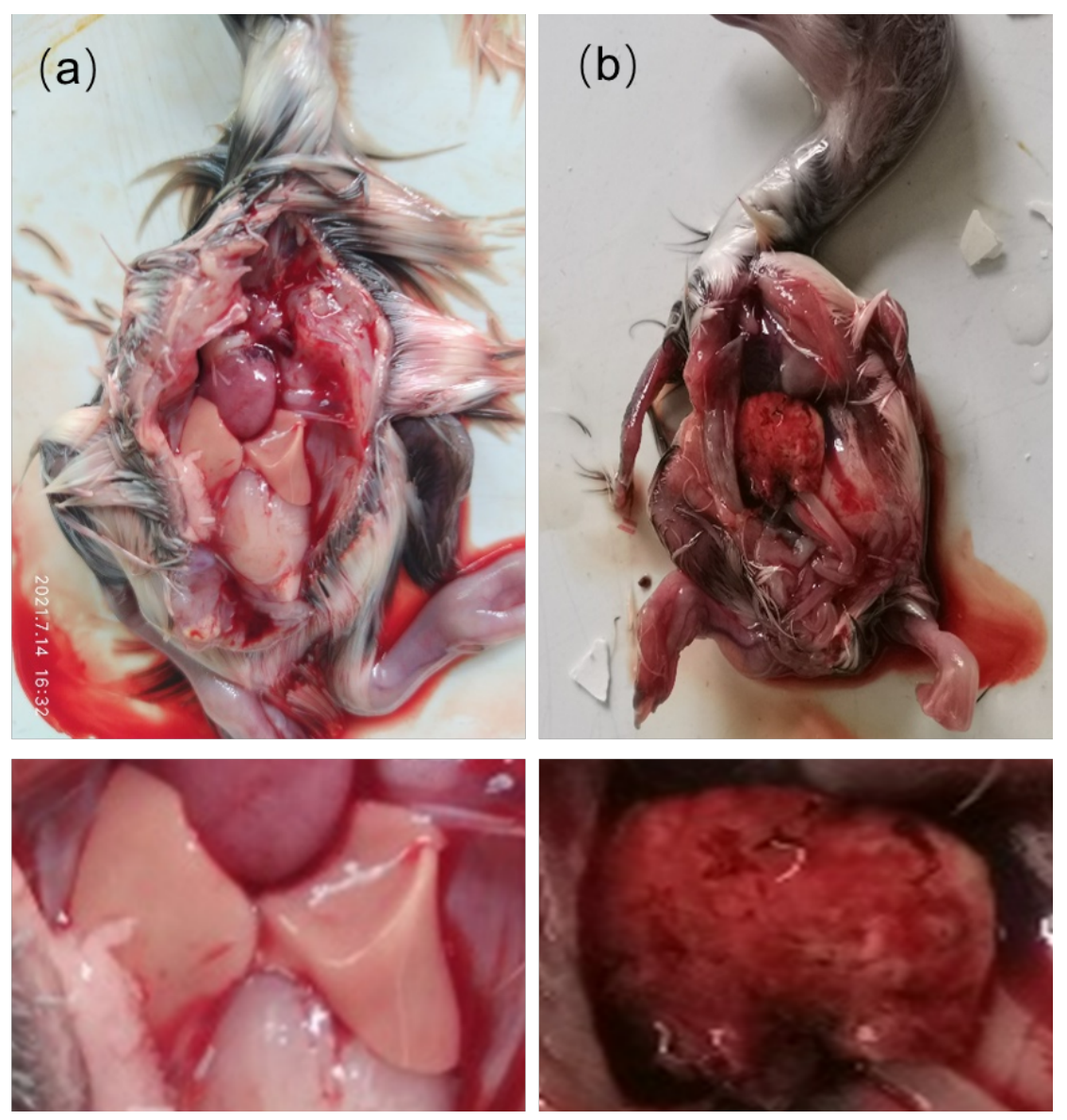

FIGURE 4 Autopsy lesions of dead embryos. (a)Liver, swollen and grayish-yellow in color, with flushed dotted lesions on the surface of the liver. (b) The liver is brittle and soft, extremely swollen and dark red, with distinct grayish-white mottled lesions on the surface of the liver.

To verify that GHPV is vertically transmitted, the isolated GHPV viral fluid was inoculated into 14-day-old goose embryos through the chorioallantoic route, and the embryos died around 8-10 days later. Dissection of the goose embryo can be seen swollen liver (FIGURE 5). The surface of the liver can be seen as obvious grayish-white mottled(FIGURE 5 A ) or dotted lesions(FIGURE 5 B ).

Goose embryos naturally infected and artificially attacked with GHPV showed similar pathological changes. PCR testing of naturally infected and artificially attacked embryonic organs revealed that GHPV was detected in the liver, spleen, kidney and heart of embryos, and the highest levels of the virus were found in the liver. The above results suggest that GHPV can be transmitted vertically.

\begin{tabular}{ll}
\hline Primer name & Primer sequence \\
\hline GPV-F & AGACTTATCAACAACCATCAYT \\
GPV-R & TCACTTATTCCTGCTGTAG \\
GHPV-F & GGATGCTGCCTCTAATTCTA \\
GHPV-R & CGCAGTTAATCAGCTTACAA \\
GRV-F & TGAGACGCCTGACTACGATT \\
GRV-R & ATGCTTGGAGTGAGACGACT \\
GPMV-F & TGCTTTCCCGAATCACCATGAC
\end{tabular}




\begin{tabular}{ll}
\hline Primer name & Primer sequence \\
\hline GPMV-R & GTTGCAACCCCAAGAGCTACAC \\
Tembusu-F & GCCACGGAATTAGCGGTTGT \\
Tembusu-R & TAATCCTCCATCTCAGCGGTGTAG \\
AstV-F & CGCTTYGATGGNATHCC \\
AstV-R & GGYTTKACCCACATNCCRAA \\
\hline
\end{tabular}
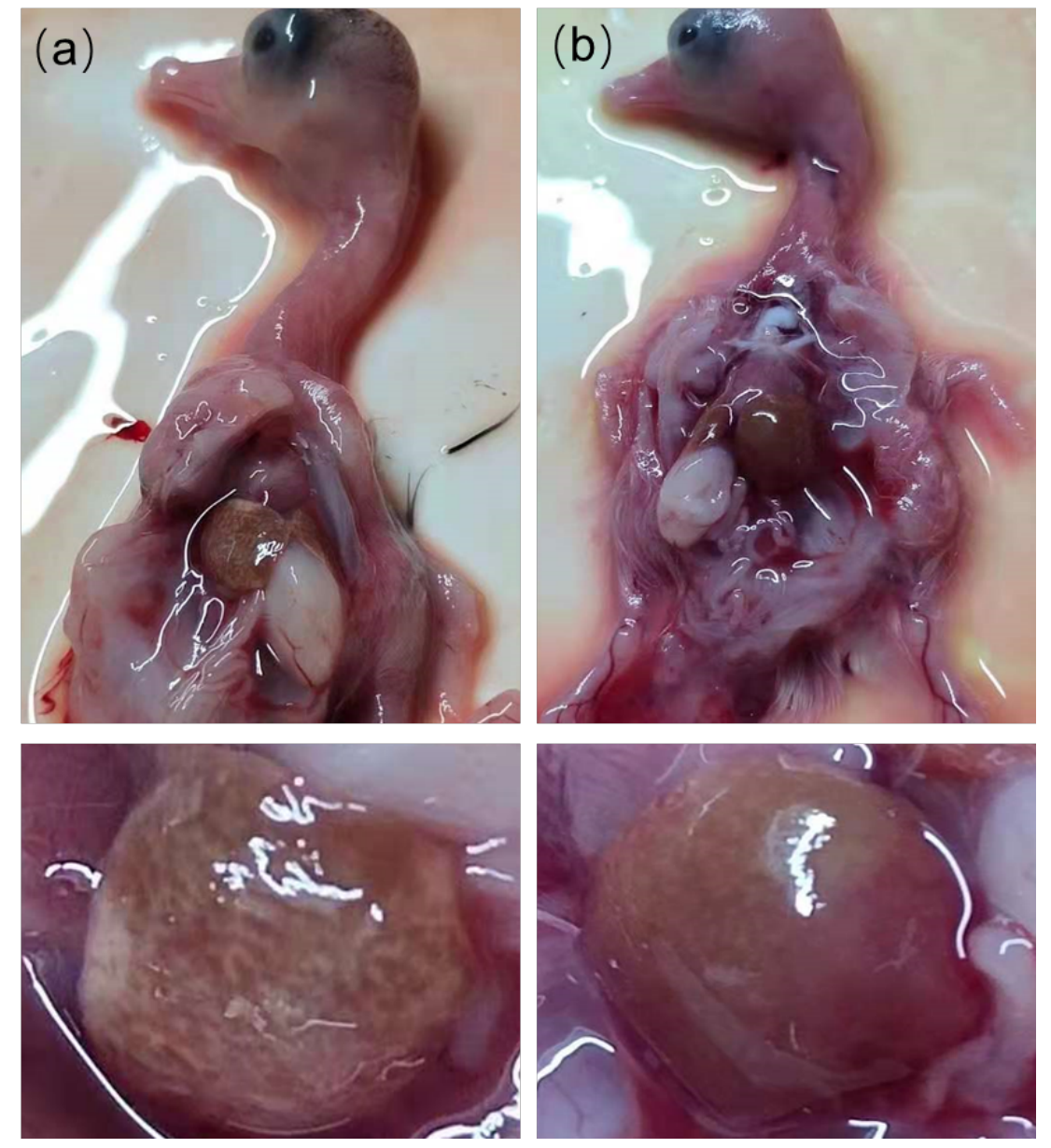

FIGURE 5 Pathological examination of embryos after attack with GHPV. The liver is swollen, and streaks(a)or corn-sized(b), grayish-white, diffusely distributed lesions are visible on the surface of the liver.

\section{Additional Documents}

Table S1 Detection Primer

Table S2 CHPV Sequencing primer

\begin{tabular}{ll}
\hline Primer name & Primer sequence \\
\hline GHPV-F1 & GATGATTGAGGTTAATTCCCTGAC \\
GHPV-R1 & ATAACAGTGTGGGTGGAAGGCAAAT \\
GHPV-F2 & GTAACCACAGTGTTGCTGGATGAGA
\end{tabular}




\begin{tabular}{ll}
\hline Primer name & Primer sequence \\
\hline GHPV-R2 & ATGAGCTTTAGAGAAATTGCAGA \\
GHPV-F3 & AGTGTACGGATCGTTGATTGACAT \\
GHPV-R3 & TCGTCTCGGTGGAGTCGGAGGT \\
GHPV-F4 & TATCTTCATAGGAGGCATGT \\
GHPV-R4 & TCTCTAACCACAATTTTCTTATC \\
GHPV-F5 & TACTATGAAGGATCTTGCACT \\
GHPV-R5 & AGCCGGTGCGGAGCCTGAAGGAGT \\
GHPV-1R & TAGAATTAGAGGCAGCATCC \\
GHPV-2F & TGTAAGCTGATTAACTGCG \\
\hline
\end{tabular}

\section{Acknowledgements}

This work was supported by the National Key Research and Development Program of China (2017YFD0500102).

\section{Ethics Statement}

This article is protected by copyright. All rights reserved

The authors confirm that no ethical approval was required as this work was carried out with collected diagnostic samples. No animal experimentation was conducted in the present study.

\section{CONFLICT OF INTEREST}

The authors declare no conflicts of interest.

\section{REFERENCES}

Bernáth, S., \& Szalai, F. (1970). Investigation for clearing the etiology of the disease appeared among goslings in 1969 (in Hungarian). Magyar Állatorvosok Lapja, 25:531-536.

Schettler, C.H. (1977). Détection en France de la néphrite hémorragique et entérite de l'oie (NHEO). [Detection of Hemorrhagic Nephritis Enteritis of Geese (HNEG) in France]. Recueil de Medecine Vetérinaire, 153:353-355.

Vuillaume, A., Tournut, J., \& Banon, H. (1982). A propos de la maladie des oisons d'apparition tardive ou néphrite hémorrhagique-entérite de l'oie (N.H.E.O.) [About the late disease onset in goslings or goose hemorrhagic enteritis nephritis (HNEG)]. Revue de Médecine Vétérinaire, 133:341-346.

Schettler C.H. (1980). Clinical picture and pathology of haemorrhagic nephritis and enteritis in geese. Tierärztliche Praxis Kleintiere, 8:313-320.

Gaweł, A., Woźniakowski, G., Samorek-Salamonowicz, E., Kozdruń, W., Bobrek, K., Bobusia, K., \& Nowak, M. (2014). Hemorrhagic nephritis and enteritis in a goose flock in Poland - Disease Accepted ArticleThis article is protected by copyright. All rights reservedcourse analysis and characterisation of etiologic agent. Avian Diseases, 58:518-522.https://doi.org/10.1637/10845-041014-Reg.1

Kozdruń, W., Woźniakowski, G., Samorek-Salamonowicz, E., \& Czekaj, H. (2012). Viral infections in goose flocks in Poland. Polish Journal of Veterinary Sciences, 15(3):525-30.https://doi.org/10.2478/v10181-0120080-9

Garmyn, A., Verlinden, M., Bosseler, L., Adriaensen, C., \& Martel, A. (2017). Persistent Goose Hemorrhagic Polyomavirus Infection on a Belgian Goose Farm. Avian Diseases, 61(4):536538.https://doi.org/10.1637/11604-020317-Case.1 
Guerin, J.L., Gelfi, J., Dubois, L., Vuillaume, A., Boucraut-Baralon, C., \& Pingret, J.L. (2000). A novel polyomavirus (goose hemorrhagic polyomavirus) is the agent of hemorrhagic nephritis enteritis of geese. Journal of Virology, 74(10):4523-9.https://doi.org/10.1128/JVI.74.10.4523-4529.2000

Bernath, S.,Farsang, A.,Kovacs, A.,Nagy, E.,Dobos-Kovacs, M.(2006). Pathology of goose haemorrhagic polyomavirus infection in goose embryos. Avian Pathol, 35: 49-52.

https://doi.org/ 10.1080/03079450500465759

Fehér, E., Lengyel, G., Dán, A., Farkas, S.L., \& Bányai, K. (2014). Whole genome sequence of a goose haemorrhagic polyomavirus detected in Hungary. Acta Microbiologica et Immunologica Hungarica, 61:221227.https://doi.org/10.1556/AMicr.61.2014.2.11

Johne, R., \& Müller, H. (2003). The genome of goose hemorrhagic polyomavirus, a new member of the proposed subgenus Avipolyomavirus. Virology, 308:291-302.https://doi.org/10.1016/S0042-6822(02)001034

Corrand, L., Gelfi, J., Albaric, O., Etievant, M., Pingret, J.L., \& Guerin, J.L. (2011). Pathological and epidemiological significance of goose haemorrhagic polyomavirus infection in ducks. Avian Pathology, 40(4):35560.https://doi.org/10.1080/03079457.2011.582481

Pingret, J.L., Boucraut-Baralon, C., \& Guérin, J.L. (2008). Goose haemorrhagic polyomavirus infection in ducks. Veterinary Record, 162(5):164.http://dx.doi.org/10.1136/vr.162.5.164-a

Dobos-Kovács, M., Horváth, E., Farsang, A., Nagy, E., Kovács, A., Szalai, F., \& Bernáth, S. (2005). Haemorrhagic nephritis and enteritis of geese: pathomorphological investigations and Accepted ArticleThis article is protected by copyright. All rights reservedproposed pathogenesis. Acta Veterinaria Hungarica, 53(2):21323.https://doi.org/10.1556/AVet.53.2005.2.6

Lacroux, C., Andreoletti, O., Payre, B., Pingret, J.L., Dissais, A., \& Guerin, J,J. (2004). Pathology of spontaneous and experimental infections by goose haemorrhagic polyoma virus. Avian Pathology, 33:351358.https://doi.org/10.1080/0307945042000220525

Chunhe, W., Cuiteng, C., Longfei, C., Rongchang, L., Guanghua, F., Shaohua, S., .. Yu, H. (2018). Genomic analysis of Sheldrake origin goose hemorrhagic polyomavirus, China. Journal of Veterinary Science, 19(6):782787.http://dx.doi.org/10.4142/jvs.2018.19.6.782

Garmyn, A., Verlinden, M., Bosseler, L., Adriaensen, C., \& Martel, A. (2017). Persistent Goose Hemorrhagic Polyomavirus Infection on a Belgian Goose Farm. Avian Diseases, 61(4):536538.https://doi.org/10.1637/11604-020317-Case.1

Kozdruń, W., Woźniakowski, G., Samorek-Salamonowicz, E., \& Czekaj, H. (2012). Viral infections in goose flocks in Poland. Polish Journal of Veterinary Sciences, 15(3):525-30.https://doi.org/10.2478/v10181-0120080-9

Leon, O., Corrand, L., Bich, T.N., Le Minor, O., Lemaire, M., Guérin, J.L., 2013. Goose Hemorrhagic polyomavirus detection in geese using real-time PCR assay. Avian Dis. 57(4):797-9. https://doi.org/10.1637/10513021013-ResNote.1

Palya, V., Ivanics, E., Glávits, R., Dán, A., Mató, T., \& Zarka, P. (2004). Epizootic occurrence of haemorrhagic nephritis enteritis virus infection of geese. Avian Pathology, 33:244250.https://doi.org/10.1080/0307945042000195740

Wan, C., Cheng, L., Fu, G., Chen, C., Liu, R., Shi, S., ...Huang, Y. (2018). Rapid detection of goose hemorrhagic polyomavirus using TaqMan quantitative real-time PCR. Molecular and Cellular Probes, 39:6164.https://doi.org/10.1016/j.mcp.2018.04.003 
Kaszab, E.,Marton, S.,Dan, A.,Farsang, A.,Balint, A.,Banyai, K.,Feher, E.(2020). Molecular epidemiology and phylodynamics of goose haemorrhagic polyomavirus. Transbound Emerg Dis,67: 26022608.https://doi.org/10.1111/tbed.13608

Tu, Y. C.,Li, W. T.,Lee, F.,Huang, C. W.,Chang, J. C.,Hsu, W. C.,Hu, S. C.,Chiou, C. J.,Chen, Y. P.(2021) Localization of goose haemorrhagic polyomavirus in naturally infected geese using in situ hybridization. Avian Pathol, 50: 41-51.https://doi.org/10.1080/03079457.2020.1832199

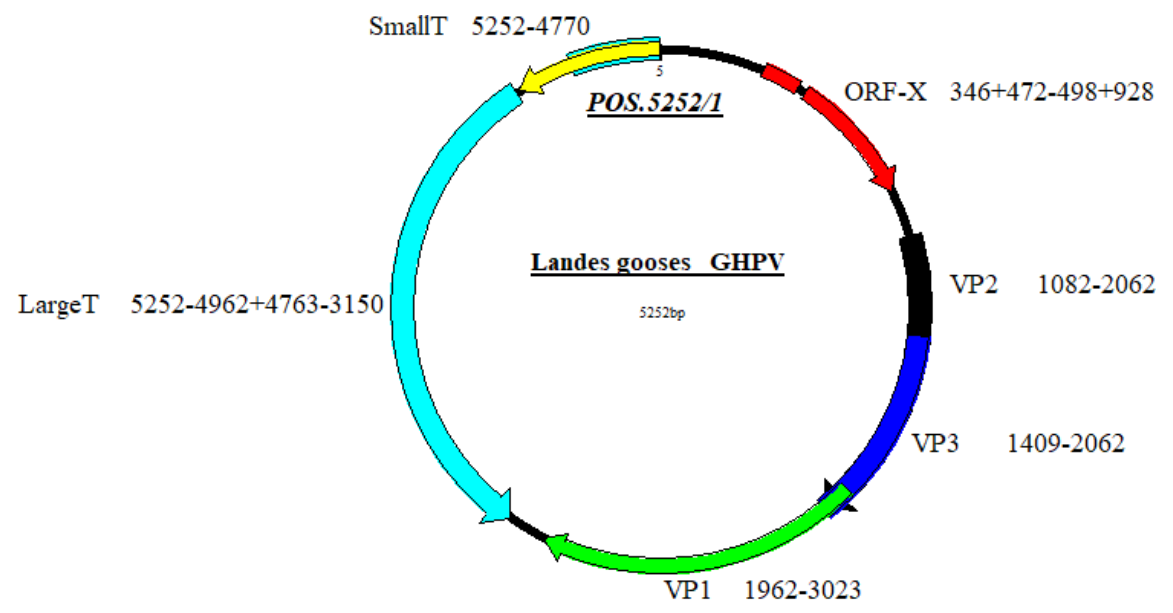

FIGURE 1
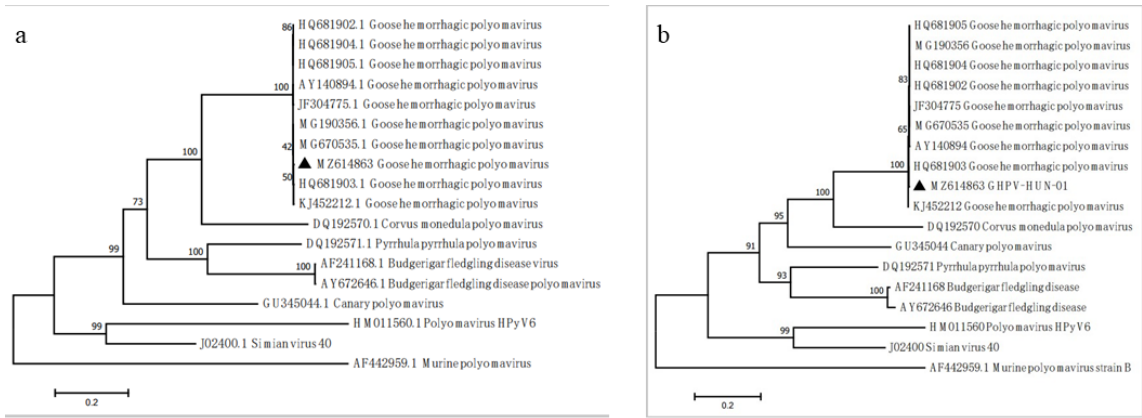

FIGURE 2 Evolutionary relationships. Evolutionary history was inferred using.
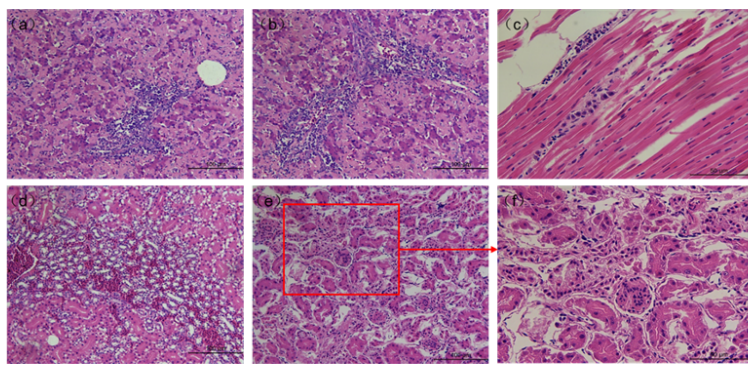

FIGURE 3 Histopathological analysis (haematoxylin and eosin staining) of tissues from a dead goose infected with GHPV. (a) A pale pink amyloid deposition in the hepatic
sinusoid widens the sinusoidal gap, and a focal infiltrate of lymphocyte-dominated mononuclear cells is seen next to the central vein. (original magnific ation 200x). Scale bar sinusoid widens the sinusoidal gap, and a focal infiltrate of lymphocyte-dominated mononuclear cells is seen next to the central vein. (original magnific ation 200x). Scale bar
indicates $100 \mu \mathrm{m}$. (b) Lymphocyte-dominated mononuclear cells show peritubular infiltration in the confluent area between liver lobules (original magnification 200x). Scale bat indicates $100 \mu \mathrm{m}$. (b) Lymphocyte-dominated mononuclear cells show peritubular infiltration in the confluent area between liver lobules. (original magnification 200x). Scale bar
indicates $100 \mu$. (c) Myocardial fibers were broken and lysed, and focal infiltration of inflammatory cells dominated by lymphocytes and macrophages was seen in the interstitium. indicates $100 \mu \mathrm{m}$. (c) Myocardial fibers were broken and lysed, and focal infilitration of inflammatory cells dominated by lymphocytes and macrophages was seen in the interstitium.
(original magnification $400 \mathrm{x}$ ). Scale bar indicates $50 \mu \mathrm{m}$.(d) Erythrocytes flow out of the blood vessels and fill the renal interstitium. (original magnific ation $200 \mathrm{x}$ ). Scale bar indicates $100 \mu \mathrm{m}$. (e) Large tubular necrosis. (original magnification 200x). Scale bar indicates $100 \mu \mathrm{m}$. (f) Renal tissues with lesions of renal tubular epithelial cells exhibited varying degrees of nuclear necrosis of renal tubular epithelial cells. (orignal magnification $400 x$ ). Scale bar indicates $50 \mu \mathrm{m}$ 

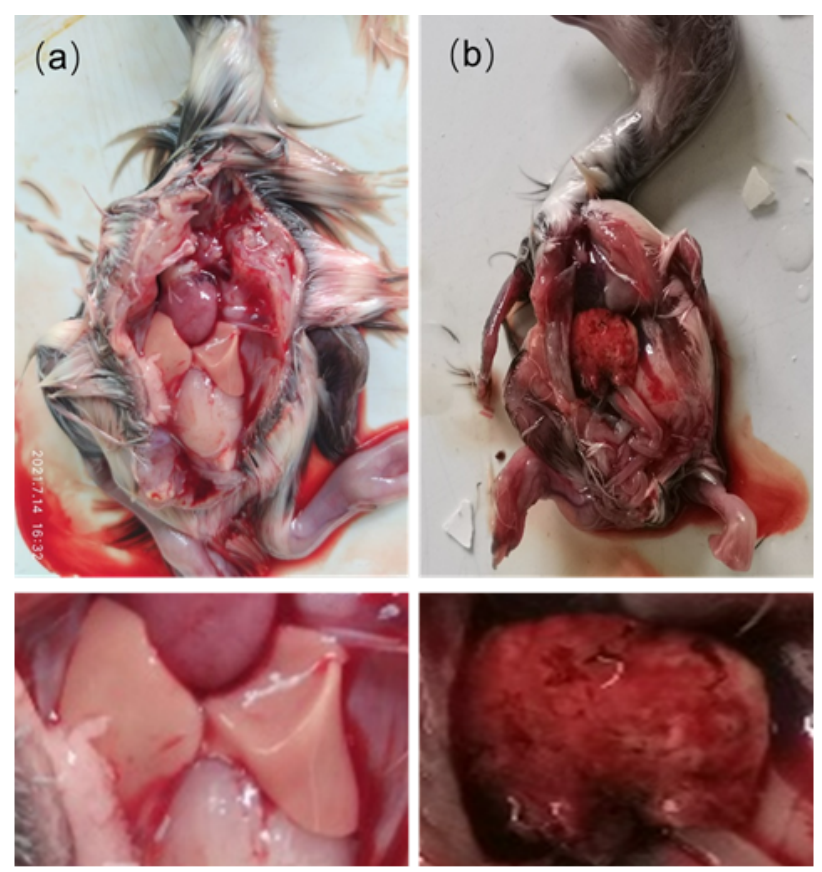

FIGURE 4 Autopsy lesions of dead embryos. (a)Liver, swollen and grayishyellow in color, with flushed dotted lesions on the surface of the liver. (b) The liver is brittle and soft, extremely swollen and dark red, with distinct grayishwhite mottled lesions on the surface of the liver.

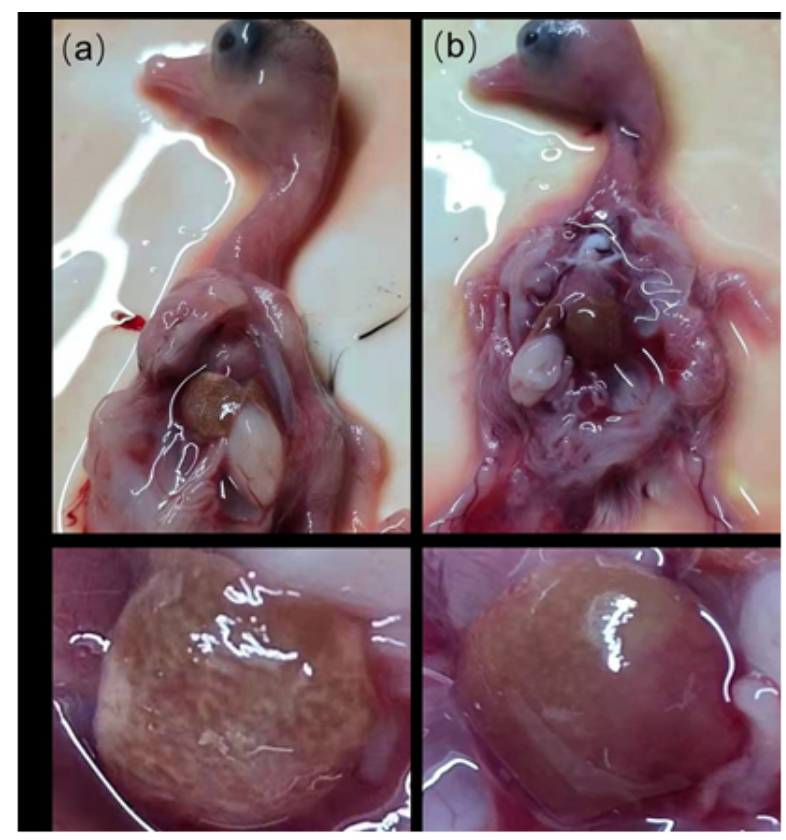

FIGURE 5 Pathological examination of embryos after attack with GHPV. The liver is swollen, and streaks (a) or corn-sized(b), grayish-white, diffusely distributed lesions are visible on the surface of the liver. 


\section{Hosted file}

Table 1. Information of the polyomaviruses strains used in this study.pdf available https://authorea.com/users/435517/articles/538483-genomic-analysis-of-landes-gooseorigin-goose-hemorrhagic-polyomavirus-china

Table S1 Detection Primer

\begin{tabular}{cc} 
& Table S1 Detection Primer \\
\hline Primer name & Primer sequence \\
\hline GPV-F & AGACTTATCAACAACCATCAYT \\
GPV-R & TCACTTATTCCTGCTGTAG \\
GHPV-F & GGATGCTGCCTCTAATTCTA \\
GHPV-R & CGCAGTTAATCAGCTTACAA \\
GRV-F & TGAGACGCCTGACTACGATT \\
GRV-R & ATGCTTGGAGTGAGACGACT \\
GPMV-F & TGCTTCCCGATCACCATGAC \\
GPMV-R & GTTGCAACCCCAAGAGCTACAC \\
Tembusu-F & GCCACGGAATTAGCGGTTGT \\
Tembusu-R & TAATCCTCCATCTCAGCGGTGTAG \\
AstV-F & CGCTTYGATGGNATHCC \\
AstV-R & GGYTTKACCCACATNCCRAA \\
\hline
\end{tabular}


Table S2 CHPV Sequencing primer

\begin{tabular}{cc}
\hline Primer name & Primer sequence \\
\hline GHPV-F1 & GATGATTGAGGTTAATCCCTGAC \\
GHPV-R1 & ATAACAGTGTGGGTGGAAGGCAAAT \\
GHPV-F2 & GTAACCACAGTGTTGCTGGATGAGA \\
GHPV-R2 & ATGAGCTTTAGAGAAATTGCAGA \\
GHPV-F3 & AGTGTACGGATCGTTGATTGACAT \\
GHPV-R3 & TCGTCTCGGTGGAGTCGGAGGT \\
GHPV-F4 & TATCTTCATAGGAGGCATGT \\
GHPV-R4 & TCTCTAACCACAATTTCTTATC \\
GHPV-F5 & TACTATGAAGGATCTTGCACT \\
GHPV-R5 & AGCCGGTGCGGAGCCTGAAGGAGT \\
GHPV-1R & TAGAATTAGAGGCAGCATCC \\
GHPV-2F & TGTAAGCTGATTAACTGCG \\
\hline
\end{tabular}

